\title{
Correction: Optimizing Text Messages to Promote Engagement With Internet Smoking Cessation Treatment: Results From a Factorial Screening Experiment
}

\author{
Amanda L Graham ${ }^{1,2}, \mathrm{PhD}$; George D Papandonatos ${ }^{3}, \mathrm{PhD} ;$ Megan A Jacobs ${ }^{1}, \mathrm{MPH}$; Michael S Amato ${ }^{1,2}, \mathrm{PhD} ;$ Sarah \\ $\mathrm{Cha}^{1}$, MSPH; Amy M Cohn ${ }^{4}$, PhD; Lorien C Abroms ${ }^{5}$, PhD; Robyn Whittaker ${ }^{6}$, PhD \\ ${ }^{1}$ Innovations Center, Truth Initiative, Washington, DC, United States \\ ${ }^{2}$ Mayo Clinic College of Medicine and Science, Rochester, MN, United States \\ ${ }^{3}$ Center for Statistical Sciences, Brown University, Providence, RI, United States \\ ${ }^{4}$ Oklahoma Tobacco Research Center, University of Oklahoma Health Sciences Center, Oklahoma City, OK, United States \\ ${ }^{5}$ Department of Prevention and Community Health, Milken Institute School of Public Health, The George Washington University, Washington, DC, \\ United States \\ ${ }^{6}$ National Institute for Health Innovation, University of Auckland, Auckland, New Zealand
}

\section{Corresponding Author:}

Amanda L Graham, PhD

Innovations Center

Truth Initiative

900 G Street, NW

4th Floor

Washington, DC, 20001

United States

Phone: 112024545938

Email: agraham@truthinitiative.org

\section{Related Article:}

Correction of: https://www.jmir.org/2020/4/e17734/

(J Med Internet Res 2020;22(7):e21027) doi: 10.2196/21027

The authors of "Optimizing Text Messages to Promote Engagement With Internet Smoking Cessation Treatment: Results From a Factorial Screening Experiment" (J Med Internet Res 2020;22(4):e17734) noticed several errors in their published manuscript which had been introduced after proofreading. The following corrections have been implemented:

The symbols $\mu$ and $\phi$ were presented as $\mathrm{m}$ and $\mathrm{f}$, respectively, in the following sentence of the Methods section; additionally, in this section, the negative sign was incorrectly subscripted:

SMDs for frequency counts were calculated as $\left(m_{1-} m_{2}\right) /\left[f\left(m_{1}+m_{2}\right)\right]^{1 / 2}$, where $m_{1}$ and $m_{2}$ were the sample means of each comparison group.

This has been revised to:

SMDs for frequency counts were calculated as $\left(\mu_{1}-\mu_{2}\right) /\left[\phi\left(\mu_{1}+\mu_{2}\right)\right]^{1 / 2}$, where $\mu_{1}$ and $\mu_{2}$ were the sample means of each comparison group.

In the same paragraph, the negative sign was again incorrectly subscripted in the following sentence:

SMDs for binary outcomes were calculated as $\left(p_{1-} p_{2}\right) /\left[p_{1} \times q_{1}+p_{2} \times q_{2}\right]^{1 / 2}$, where $p_{1}=1-q_{1}$ and $p_{2}=1-q_{2}$ were the sample outcome prevalence of each
comparison group.

This has been revised to:

SMDs for binary outcomes were calculated as $\left(p_{1}-p_{2}\right) /\left[p_{1} \times q_{1}+p_{2} \times q_{2}\right]^{1 / 2}$, where $p_{1}=1-q_{1}$ and $p_{2}=1-q_{2}$ were the sample outcome prevalence of each comparison group.

Additionally, due to a technical error the following sentence was published in the Results section of the Abstract:

As no SMD \&gt;0.30 was observed for main effects on any outcome, results suggest that for some outcomes, the combined intervention was stronger than individual factors alone.

This has been revised to:

As no SMD >0.30 was observed for main effects on any outcome, results suggest that for some outcomes, the combined intervention was stronger than individual factors alone.

The correction will appear in the online version of the paper on the JMIR website on July 28, together with the publication of this correction notice. Because this was made after submission 
to PubMed, PubMed Central, and other full-text repositories, repositories. the corrected article has also been resubmitted to those

This is a non-peer-reviewed article. Submitted 03.06.20; accepted 03.06.20; published 28.07.20.

Please cite as:

Graham AL, Papandonatos GD, Jacobs MA, Amato MS, Cha S, Cohn AM, Abroms LC, Whittaker R

Correction: Optimizing Text Messages to Promote Engagement With Internet Smoking Cessation Treatment: Results From a Factorial Screening Experiment

J Med Internet Res 2020;22(7):e21027

URL: https://www.jmir.org/2020/7/e21027

doi: $\underline{10.2196 / 21027}$

PMID: 32721924

(C)Amanda L Graham, George D Papandonatos, Megan A Jacobs, Michael S Amato, Sarah Cha, Amy M Cohn, Lorien C Abroms, Robyn Whittaker. Originally published in the Journal of Medical Internet Research (http://www.jmir.org), 28.07.2020. This is an open-access article distributed under the terms of the Creative Commons Attribution License (https://creativecommons.org/licenses/by/4.0/), which permits unrestricted use, distribution, and reproduction in any medium, provided the original work, first published in the Journal of Medical Internet Research, is properly cited. The complete bibliographic information, a link to the original publication on http://www.jmir.org/, as well as this copyright and license information must be included. 